\title{
Erratum to: LapOntoSPM: an ontology for laparoscopic surgeries and its application to surgical phase recognition
}

\author{
Darko Katic $^{1}$ - Chantal Julliard ${ }^{1}$ - Anna-Laura Wekerle ${ }^{2}$ - Hannes Kenngott ${ }^{2}$ . \\ Beat Peter Müller-Stich ${ }^{2}$ - Rüdiger Dillmann ${ }^{1}$. Stefanie Speidel ${ }^{1}$. \\ Pierre Jannin $^{3,4}$ - Bernard Gibaud ${ }^{3,4}$
}

Published online: 24 December 2015

(C) CARS 2015

\section{Erratum to: Int J CARS (2015) 10:1427-1434 DOI 10.1007/s11548-015-1222-1}

The original version of this article unfortunately contained a mistake in the affiliation. The correct affiliations are

Darko Katić, Chantal Julliard, Rüdiger Dillmann and Stefanie Speidel

Humanoids and Intelligence Systems Lab, Institute for Anthropomatics and Robotics, Karlsruhe Institute of Technology (KIT), 76131 Karlsruhe, Germany
Anna-Laura Wekerle, Hannes Kenngott and Beat Peter Müller-Stich

Department of General, Visceral and Transplant Surgery, University of Heidelberg, 69120 Heidelberg, Germany

Pierre Jannin and Bernard Gibaud

INSERM, UMR 1099, 35000 Rennes, France

Pierre Jannin and Bernard Gibaud

Université de Rennes 1, LTSI, 35000 Rennes, France

The online version of the original article can be found under doi:10.1007/s11548-015-1222-1.

Darko Katić

katic@kit.edu

1 Humanoids and Intelligence Systems Lab, Institute for Anthropomatics and Robotics, Karlsruhe Institute of Technology (KIT), 76131 Karlsruhe, Germany

2 Department of General, Visceral and Transplant Surgery, University of Heidelberg, 69120 Heidelberg, Germany

3 INSERM, UMR 1099, 35000 Rennes, France

4 Université de Rennes 1, LTSI, 35000 Rennes, France 